\title{
Berliner Ophthalmologische Gesellschaft
}

\section{Sitzung vom 17. Dezember 1908}

Vorsitzender: Herr v. Michel. Schriftführer: Herr Wertheim.

Herr Leber: Untersuchungen zur Aetiologie des Trachoms.

Der Vortr. zeigt in zahlroichen Abbildung $\beta n$ die bekannten Körnchen in den Epithelzellen Trachomatöser, die von einigen Autoren (Halberstädter, Prowatschek. Greeff, Clausen)) als Parasiten angesehen und mit der Trachom-ätiologi $\beta$ in Verbindung gebracht werden. A. Leber hat die Untßrsuehungs-mothod $\theta$ insoweit geändert, als er zur Fixierung die feuchte Sublimatfixierung anwendete, die Schaudinn für seine Protozoen-Untersuchungen benutzte; neu ist ferner die Angabe, dass er die Körnchen in od $\beta r$ auf roten Blutkörperehen gefundon habe. Auch Herr G. di Santo demonstrierte Trachomhörperchen im Schnitt und wies mit seinen Untersuchungen an Affen und Menschen nach, dass die Körperchen sich auch im subkonjunktivalen Bindeg $\theta$ webe fänden.

Unfall- und Versicherungskunde.

83

Dískussion.

Herr Herzog meint, dass die gezeigton G $\theta$ bilde zu wenig different von solchen seien, die bei einfachen chronischen Entzündungen vorkommen, und zeigt Abbildungen, die eine grosse Aehnlichkeit mit ,,Trachomkörnern" haben.

In der Diskussion über den Vortrag des Herrn Hertzell (cf. vorige Sitzung) antwortet dieser auf die $\Gamma$ rage, ob bereits mit der Photographie des durch -1ßucht $\theta$ ten Augenhintergrundes Resultate erzielt seien, dass die Photographie prinzipiell als möglich zu bezeiehnen sei, da das Bild vom Fundus sich präzisieren lasse und die Reflexe fehlen. Die Expositionsdauer dürfte wohl abßr eine längere sein müssen.

Herr Loser: Das Verhalten der Sehschärfe in farbigem Licht. Loser hat Untersuchungen der Sehschärfe in weissem, rotem und grünem Licht von gleicher Helligkeit angestellt. Die Helligkeitsmessung der verschiedenfar big-en. Lichter, die bei derartigen Versuchen eine gewisse Schwierigkeit darbietet, wurde mittels der sog. „Flimmer-Photometrie” vorgenommen. Es ergab sich, dass die Sehschärfe für ,,Rot” viel geringer ist als für ,, Weiss” und ,,Grün”, während zwischen diesen beiden Farben kein Unterschied besteht. In weiteren Versuchen wurde die ursprünghche Lichtintensität mittels des Aubertschen Episcopisters auf \%, bezw. ' /16 bezw. $>$ /as herabgesetzt. Dabei zeigte sich, dass der zwischen Rot einerseits und Weiss und Grün anderseits bestehende Unterschied entsprechend der Abnahme der Lichtintensität immer mehr sich verminderte bezw. naliezu verschwand. Daraus muss geschlossen werden, dass das geschilderte Verhalten nicht durch den farbigen Anted der Lichter bedingt sein kann, sondern auf andere, wohl kompliziertere Faktoren zu beziehen ist. Vor allem kommt hier das in Be-tracht, was Helmholtz , Lichtstärke grösster Klarheit” oder , ,klarste Be-1ßuchtung” genannt hat, und L. halt es für wahrscheinlich, dass diesem Maximum der Klarheit für Rot eine andere objektive Lichtstärke entspricht als für Grün. Deshalb halt L. die Schlussfolgerungen, die von mancher Seite aus derartigen Sehschärfebestimmungen in weissem bezw. farbigem Lichte gezogen 
wurden und die sich sowohl nach der praktischen wie nach der theorßtischen Seite hin erstrecken, für viel zu weitgehend und nicht ge-nügend gestützt. (Autorefsrat.)

Herr A dam stellt einen Fall von Keübeinsarkom vor, wo bei f olgende Sym-ptome vorhanden waren: R. Abduconsparese (vorübergehend hatte angeblich Amaurosebestanden), L.

Exophthalmus, Ptosis, Unbeweglichkeit des Bulbus, Amaurose. Fundus normal. Weiter bestand Lähmung des linken motori-schen Trigeminus, des I. Astes des sensiblen Trigeminus, des r.

Gaumen-segels, des rechten Hypoglossus und Störungen im Bereich des rechten Aeusticus. An der rechten Halsseite fanden sich grosse Drüsenpackete.

Rhinologisch wurde im Nasen-Rachenraum ein Tumor konstatiert, der scheinbar mit der Keilbeinhöhle in Verbindung stand, da man mit derSonde keinen knöchem $\beta$ n Widerstand fühlt $\theta$. Die Lähmungen des Gaumen-segels, Hypoglossus, motorischen Trigeminus finden ihre Erklärungen durchden Tumor und seine Metastasen. Die Hörstörung ist auf einen Míttelohrkatarrh (Tuba Eustachii) zurückzuführen. Der Tumor selbst hat wohl dasKeilbein nach don Seiton durchbrochen und ist auf dem Wege der Fiss \uraorbitalis superior in die Augenhöhle hineingewuchert, womit die Symptomeerklärlich werden. Pollack.

Unfall- und Versicherungskunde. 